\title{
Manipulating the Net Radiative Recombination Rate in Lead Halide Perovskite Films by Modification of Light Outcoupling
}

\author{
Florian Staub ${ }^{*}, 1$, Thomas Kirchartz, ${ }^{*}, 1,2$ Karsten Bittkau ${ }^{1}$, Uwe Rau $^{1}$ \\ ${ }^{1}$ IEK-5 Photovoltaik, Forschungszentrum Jülich GmbH, 52425 Jülich, Germany \\ ${ }^{2}$ Faculty of Engineering and CENIDE, University of Duisburg-Essen, Carl-Benz-Str. 199, 47057 \\ Duisburg, Germany
}

\section{AUTHOR INFORMATION}

\section{Corresponding Author}

*t.kirchartz@fz-juelich.de

\section{ORCID}

Florian Staub: 0000-0002-0524-8262

Thomas Kirchartz: 0000-0002-6954-8213

Karsten Bittkau: 0000-0001-5381-2967

Uwe Rau: 0000-0003-3526-3081

\section{Notes}

The authors declare no competing financial interests. 


\section{ABSTRACT}

Photon recycling is a fundamental physical process that becomes especially important for photovoltaic devices that operate close to the radiative limit. This implies that the externally measured radiative decay rate deviates from the internal radiative recombination rate of the material. In the present paper, the probability of photon recycling in organic lead halide perovskite films is manipulated by modifying the underlying layer stacks. We observe recombination kinetics by time-resolved photoluminescence that are controlled by the optical design of the chosen layer structure. Quantitative simulations of decay rates and emission spectra show excellent agreement with experimental results if we assume that the internal bimolecular recombination coefficient is $\sim 66 \%$ radiative.

\section{TOC GRAPHIC}

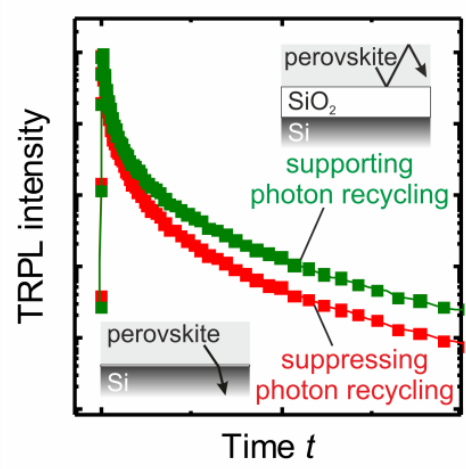


The radiative recombination rate describing the interaction of photons and electronic charge carriers of a photovoltaic absorber material is the materials' most fundamental and relevant physical property with respect to its photovoltaic action. Because of the principle of detailed balance the recombination rate is quantitatively linked to the absorption coefficient of the material. ${ }^{1}$ Photon recycling, i.e. the reabsorption of emitted photons by the absorber material itself, harmonizes the radiative recombination rate with the radiative emission flux from a corresponding solar cell. ${ }^{2}$ Photon recycling is thus the fundamental physical link between the bulk properties of the absorber material and the inevitable radiative losses from the solar cell to the outside world defining the radiative efficiency limit. ${ }^{3}$ The analysis of photovoltaic materials close to their radiative limit ${ }^{4-6}$ therefore requires a careful consideration of the fate of internally generated photons. ${ }^{4,7,8}$

Organic inorganic perovskites exhibit extraordinary charge carrier lifetimes ${ }^{9,10}$ which enable high internal luminescence quantum yields. ${ }^{9,11}$ In combination with the observed large energetic overlap of luminescence emission with the absorption profile, ${ }^{12-14}$ organic inorganic perovskites are therefore a paramount system to investigate photon recycling. ${ }^{15-20}$ For photovoltaic operation, photon recycling implies the following features: (i) A fraction of charge carriers is regained after being temporarily lost due to radiative recombination. ${ }^{21}$ (ii) The obtained (external) radiative lifetime from measurements is significantly prolonged by the number of photon recycling events. ${ }^{22-24}$ When the radiative lifetimes are not corrected for photon recycling, the internal luminescence quantum efficiencies are underestimated. ${ }^{25}$ (iii) If photons created by radiative recombination within the solar cell are reabsorbed (recycled) as opposed to being parasitically absorbed, the open-circuit voltage increases..$^{26,27}$ 
Radiative recombination rates in lowly doped semiconductors like metal-halide perovskites are proportional to the square of the excess carrier density. While it is relatively easy to detect quadratic (often also referred to as bimolecular) recombination terms in transient photoluminescence, absorption or photoconductivity measurements, ${ }^{28-30}$ it is more difficult to find solid evidence that the quadratic recombination terms are indeed due to radiative recombination alone. Recent work by Richter et al., ${ }^{31}$ who combined transient spectroscopy measurements with findings from photoluminescence quantum efficiency measurements, suggested that there are also non-radiative contributions to the quadratic recombination term seen in experiments. In order to study the nature of the quadratic recombination term in more detail, in this work we make use of the sensitivity of the apparent rate constant on photon recycling and, in turn, the sensitivity of photon recycling on the amount of parasitic absorption in the sample, as described in the following.

Photons created by radiative recombination in the perovskite layer can either be emitted from the sample (with probability $p_{\mathrm{e}}$ ), be reabsorbed (with probability $p_{\mathrm{r}}$ ) or be parasitically absorbed (with probability $p_{\mathrm{a}}$ ) by other layers such as the metal contacts in a solar cell. The three probabilities have to add up to one, and therefore the amount of reabsorption that should be visible in the apparent rate constant of any radiative decay process is given by $p_{\mathrm{r}}=1-p_{\mathrm{e}}-p_{\mathrm{a}}$. Thus, any modification of e. g. the amount of parasitic absorption should affect this rate constant. Furthermore, any changes seen in the quadratic decay constant that can be modulated by a change of the amount of parasitic absorption and that cannot be attributed to any change in electronic properties will provide evidence that the quadratic recombination term is at least partly radiative. 
We therefore prepared two simple layer stacks as illustrated in Fig. 1 (see supporting information for more details). Silicon dioxide layers of distinct thicknesses (200 nm and $2.3 \mathrm{~nm}$ ) are thermally grown on a crystalline silicon wafer, and $\mathrm{CH}_{3} \mathrm{NH}_{3} \mathrm{PbI}_{3}$ perovskite films are formed on top by spin coating. The 200 nm-thick silicon dioxide layer in the stack of Fig. 1 (a) eventually causes total internal reflection of photoluminescence at the perovskite/SiO $2 \mathrm{interface}$ due to the lower refractive index of $\mathrm{SiO}_{2}\left(n_{\mathrm{r}, \mathrm{SiO}_{2}} \approx 1.4\right)^{32}$ compared to perovskite ( $n_{\mathrm{r} \text {,pero }} \approx$ $2.6)^{33}$ at photon energies around $(1.4-1.9) \mathrm{eV}$, i. e. in the range of the perovskite photoluminescence. Consequently, all photons emitted in angles larger than the critical angle of total internal reflection $\theta_{\mathrm{c}} \gtrsim 35^{\circ}$ are expected to be emitted into modes guided within the perovskite layer which are eventually reabsorbed. Waveguide modes have been observed for similar stacks. ${ }^{34}$ In the case of the $2.3 \mathrm{~nm}$-thin $\mathrm{SiO}_{2}$ layer (Fig. 1 (b)), the light reflectance is strongly suppressed through evanescent light coupling. Therefore, radiation is efficiently coupled into the silicon wafer $\left(n_{\mathrm{r}, \mathrm{Si}} \approx 3.7\right),{ }^{35}$ where it is directly absorbed. We use a thin residual layer of $\mathrm{SiO}_{2}$ to enable sufficient wetting by the perovskite solution, because solution-processed perovskites on top of bare silicon cannot be realized due to inadequate adhesion. Furthermore, electrically insulating $\mathrm{SiO}_{2}$ prevents charge transfer from perovskite into silicon, which would hamper the analysis of photoluminescence transients because the effect of charge carrier quenching on the shapes of transients is not easily unraveled. ${ }^{36-38}$ By employing $\mathrm{SiO}_{2}$ as substrate for the growth of perovskite in both stack designs, the formation of crystalline perovskite with comparable quality can be ensured, which will be confirmed below.

Both samples finish with the perovskite layer and thus have the same interface with a surrounding medium in common, which is nitrogen as an inert atmosphere for the photoluminescence measurements. For optical simulations, we assume a non-absorbing medium 
with a refractive index of $n_{\mathrm{r}}=1$, which we denote here as air. The amount of photon recycling is tuned by the underlying layer structure alone. Thus, if there is a substantial amount of radiative recombination, the structure embedding the thick silicon dioxide layer is expected to show a pronounced effect of photon recycling, while the structure with the thin silicon dioxide layer will result in a reduced amount of photon recycling on the other hand.

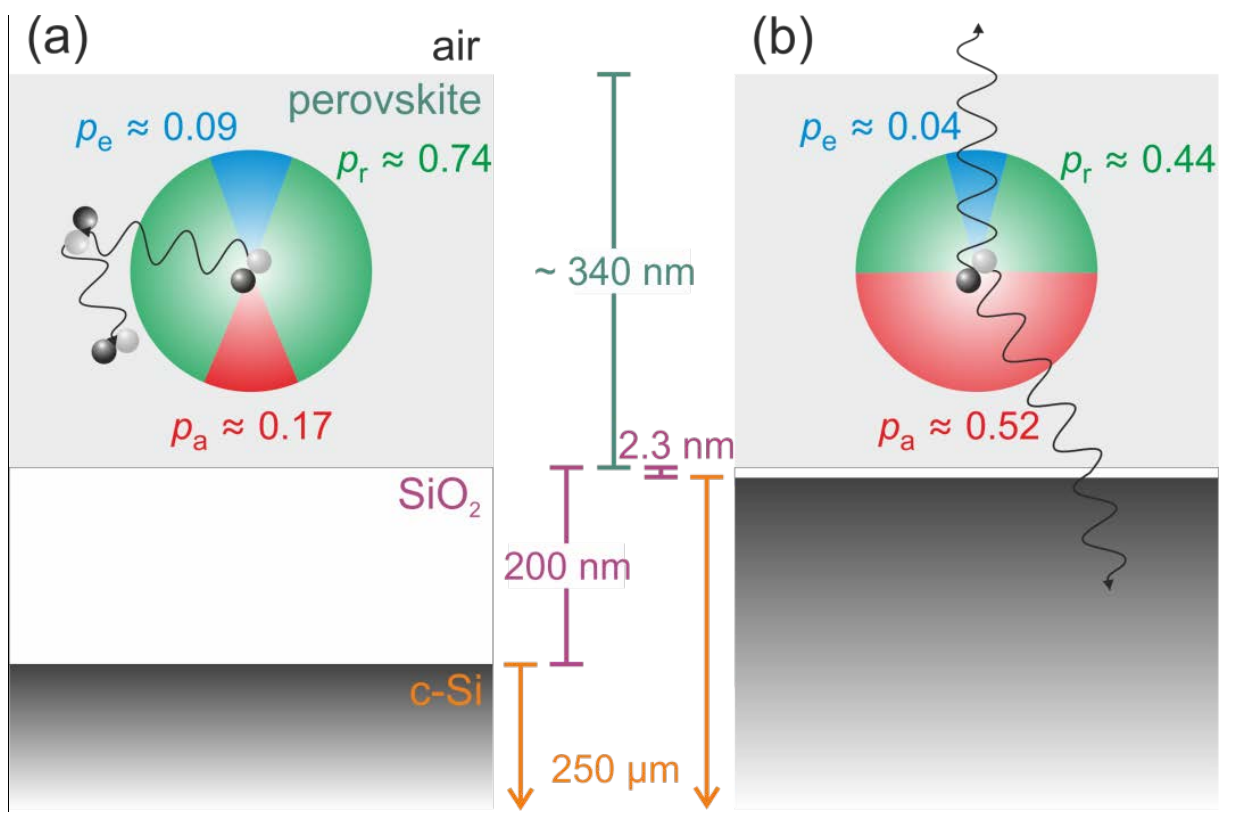

Figure 1. Schematic depiction of the experimental stack designs that allow us to manipulate reabsorption by modifying parasitic absorption. Isotropic photon emission arising from radiative electron-hole annihilation inside the perovskite layers $(\sim 340 \mathrm{~nm})$ is indicated as a circle. The colored circular segments illustrate various emission cones, which facilitate photon outcoupling (blue), parasitic photon absorption (red) by entering the crystalline silicon wafer $(250 \mu \mathrm{m})$, and photon reabsorption (green), respectively. By significantly changing the thickness of the low refractive silicon dioxide layer embedded between perovskite and crystalline silicon from $200 \mathrm{~nm}$ in (a) to $2.3 \mathrm{~nm}$ in (b), the probabilities of parasitic absorption $\left(p_{\mathrm{a}}\right)$, outcoupling $\left(p_{\mathrm{e}}\right)$ as well as of photon recycling $\left(p_{\mathrm{r}}\right)$ are tuned. The corresponding values derived from optical simulations are indicated. 
As a consequence, the corresponding externally measured radiative decay rates of the layers are expected to be different (see Eq. (3)). In order to investigate the (radiative) recombination dynamics, we show time-resolved photoluminescence (TRPL) spectroscopy measurements of the perovskite films on top of the two distinct layer stacks in Fig. 2. Here, green symbols represent the sample with the embedded $200 \mathrm{~nm}$-thick silicon dioxide layer, whereas red symbols mark the TRPL data for the stack with the $2.3 \mathrm{~nm}$-thin silicon dioxide layer. In the normalized depiction of Fig. 2 (a), the decay dynamics at low excitation fluences $\left(0.12 \mu \mathrm{J} / \mathrm{cm}^{2}\right.$, circles) are found to be equal for the two samples. As trap-assisted Shockley-Read-Hall mechanisms govern recombination upon low level injection conditions, ${ }^{25,39,28}$ the two stacks are best comparable concerning the influence of defects within the perovskite films. When higher excitation fluences are applied, the photoluminescence transients of the two stacks clearly diverge. This finding can be explained by differences in higher order recombination kinetics as one would expect from the experimental design. In order to quantify these observations, the experimental decay curves in Fig. 2 (b) are globally fitted according to the commonly applied recombination model in order to derive Shockley-Read-Hall, radiative and Auger recombination parameters. In doing so, the obtained TRPL intensity $I(t)$ is a function of the transient excesscharge carrier concentration $n(t)$ and is expressed under high level injection conditions as

$$
I(t) \propto k_{\mathrm{rad}}^{\mathrm{ext}} n(t)^{2}
$$

Here, $k_{\text {rad }}^{\text {ext }}$ denotes the externally observed radiative recombination coefficient. In order to simulate the TRPL decays, the excess-charge carrier concentration $n(t)$ has to be modeled by solving the continuity equation ${ }^{25,29}$ numerically

$$
\frac{\partial n(t)}{\partial t}=G_{\mathrm{exc}}(t)-C n(t)^{3}-k^{\mathrm{ext}} n(t)^{2}-\frac{n(t)}{\tau_{\mathrm{SRH}}},
$$


where $G_{\text {exc }}(t)$ represents the temporary generation rate due to the excitation pulse (see supporting material for more details), $C$ denotes the Auger recombination coefficient and $\tau_{\mathrm{SRH}}$ is the lifetime attributed to Shockley-Read-Hall recombination statistics. Here, $k^{\text {ext }}$ indicates the externally observed bimolecular recombination coefficient, which is the sum of $k_{\text {rad }}^{\text {ext }}$ and a potential non-radiative contribution $k_{\text {nrad }}$ as proposed by Richter et al. ${ }^{31}$

Table 1. Recombination parameters (Auger recombination coefficient $C$, external bimolecular recombination coefficient $k^{\text {ext }}$ and Shockley-Read-Hall lifetime $\tau_{\mathrm{SRH}}$ ) derived from global fits of the respective time-resolved decays obtained from two samples of the same layer sequence perovskite/ $\mathrm{SiO}_{2} / \mathrm{Si}$ but with different silicon dioxide thicknesses, namely $200 \mathrm{~nm}$ and $2.3 \mathrm{~nm}$.

\begin{tabular}{cccc}
\hline $\mathrm{SiO}_{2}$ thickness & $k^{\mathrm{ext}}\left(\mathrm{cm}^{3} \mathrm{~s}^{-1}\right)$ & $C\left(\mathrm{~cm}^{6} \mathrm{~s}^{-1}\right)$ & $\tau_{\mathrm{SRH}}(\mathrm{ns})$ \\
\hline $200 \mathrm{~nm}$ & $(4.6 \pm 0.2) \times 10^{-11}$ & $(8.0 \pm 2.1) \times 10^{-29}$ & $870 \pm 140$ \\
$2.3 \mathrm{~nm}$ & $(6.3 \pm 0.3) \times 10^{-11}$ & $(9.7 \pm 1.4) \times 10^{-29}$ & $990 \pm 150$ \\
\hline
\end{tabular}




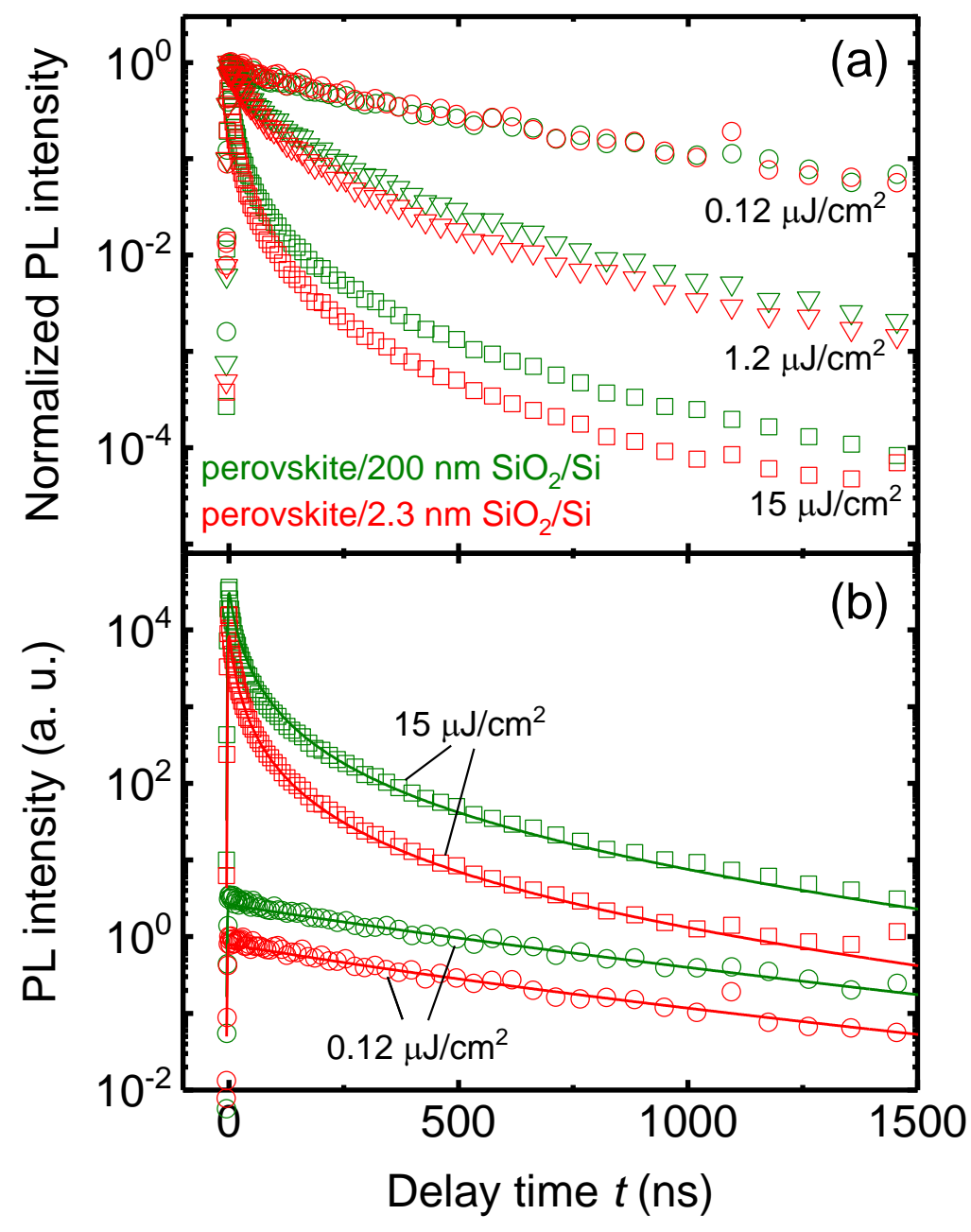

Figure 2. (a) Measured normalized time-resolved photoluminescence decays of perovskite films on top of two layer stacks with different silicon dioxide thicknesses: perovskite/200 nm SiO $2 / S i$ (green symbols) and perovskite/2.3 $\mathrm{nm} \mathrm{SiO} / \mathrm{Si}$ (red symbols). Panel (b) shows a selection of the same experimental data without normalization. Equal excitation fluences are applied for both layer stacks (15 $\mu \mathrm{J} / \mathrm{cm}^{2}$ (squares), $1.2 \mu \mathrm{J} / \mathrm{cm}^{2}$ (triangles) and $0.12 \mu \mathrm{J} / \mathrm{cm}^{2}$ (circles)). The solid lines represent fitted photoluminescence transients.

A good agreement between the globally simulated photoluminescence transients with the experimental data is demonstrated in Fig. 2 (b). The recombination parameters obtained from the 
fits are listed in Tab. 1. While the SRH lifetimes as well as the Auger recombination coefficients each coincide within the error margins, the external bimolecular recombination coefficients $k^{\text {ext }}$ vary by a factor of $k_{\text {ratio }}^{\text {ext }}=k_{2}^{\text {ext }} / k_{200}^{\text {ext }} \approx 1.4$. Here and in the following, the indices "200" and “2” of stated physical quantities relate to the layer stacks exhibiting the $200 \mathrm{~nm}$-thick and the 2.3 nm-thin silicon dioxide layer, respectively. The external radiative recombination coefficient $k_{\text {rad }}^{\text {ext }}$ is reduced with respect to the internal radiative recombination coefficient $k_{\text {rad }}^{\text {int }}$ due to photon recycling events ${ }^{25}$

$$
k_{\mathrm{rad}}^{\mathrm{ext}}=\left(1-p_{\mathrm{r}}\right) k_{\mathrm{rad}}^{\mathrm{int}}
$$

For the investigated samples, $k_{2}^{\text {ext }}$ is found to be 1.4 times larger than $k_{200}^{\text {ext }}$, which, according to Eq. (3), we can directly relate to a smaller probability $p_{\mathrm{r}, 2}$ of photon recycling compared to $p_{\mathrm{r}, 200}$. Taking a non-radiative bimolecular contribution $k_{\mathrm{nrad}}$ into account, we find

$$
k_{\mathrm{ratio}}^{\mathrm{ext}}=\frac{k_{\mathrm{rad}, 2}^{\mathrm{ext}}+k_{\mathrm{nrad}}}{k_{\mathrm{rad}, 200}^{\mathrm{ext}}+k_{\mathrm{nrad}}}=\frac{\left(1-p_{\mathrm{r}, 2}\right) k_{\mathrm{rad}}^{\mathrm{int}}+k_{\mathrm{nrad}}}{\left(1-p_{\mathrm{r}, 200}\right) k_{\mathrm{rad}}^{\mathrm{int}}+k_{\mathrm{nrad}}}=\frac{\left(1-p_{\mathrm{r}, 2}\right) \kappa+1-\kappa}{\left(1-p_{\mathrm{r}, 200}\right) \kappa+1-\kappa}=\frac{1-p_{\mathrm{r}, 2} \kappa}{1-p_{\mathrm{r}, 200} \kappa} .
$$

Here, $k_{\text {rad }}^{\text {int }}$ is assumed to be an intrinsic material property and, thus, is considered to be identical in both perovskite layers. As the origin of $k_{\mathrm{nrad}}$ remains unknown, it is not clear whether $k_{\mathrm{nrad}}$ can also be treated as a material-specific, intrinsic parameter. Besides, $k_{\text {nrad }}$ could be coupled to defect-related recombination in the sample. However, as similar trap-assisted Shockley-ReadHall lifetimes are obtained for both stacks, also $k_{\text {nrad }}$ should be rather identical in this scenario. For the last two terms in Eq. (4), we made use of the definition of the radiative fraction

$$
\kappa=\frac{k_{\mathrm{rad}}^{\mathrm{int}}}{k_{\mathrm{rad}}^{\mathrm{int}}+k_{\mathrm{nrad}}}
$$

Solving Eq. (4) for $\kappa$, finally yields 


$$
\kappa=\frac{k_{\mathrm{ratio}}^{\mathrm{ext}}-1}{p_{\mathrm{r}, 200} k_{\mathrm{ratio}}^{\mathrm{ext}}-p_{\mathrm{r}, 2}}
$$

Thus, with the knowledge of the experimental ratio $k_{\text {ratio }}^{\text {ext }} \approx 1.4$ and the $p_{\mathrm{r}}$ values of both samples, we are not only able to verify the existence of a non-radiative bimolecular recombination channel but also estimate the fraction $\kappa$ of radiative, quadratic recombination. Figure 3 illustrates Eq. (6) and shows which radiative fraction $\kappa$ can be expected for a combination of possible photon recycling probabilities $p_{\mathrm{r}, 2}$ and $p_{\mathrm{r}, 200}$. 


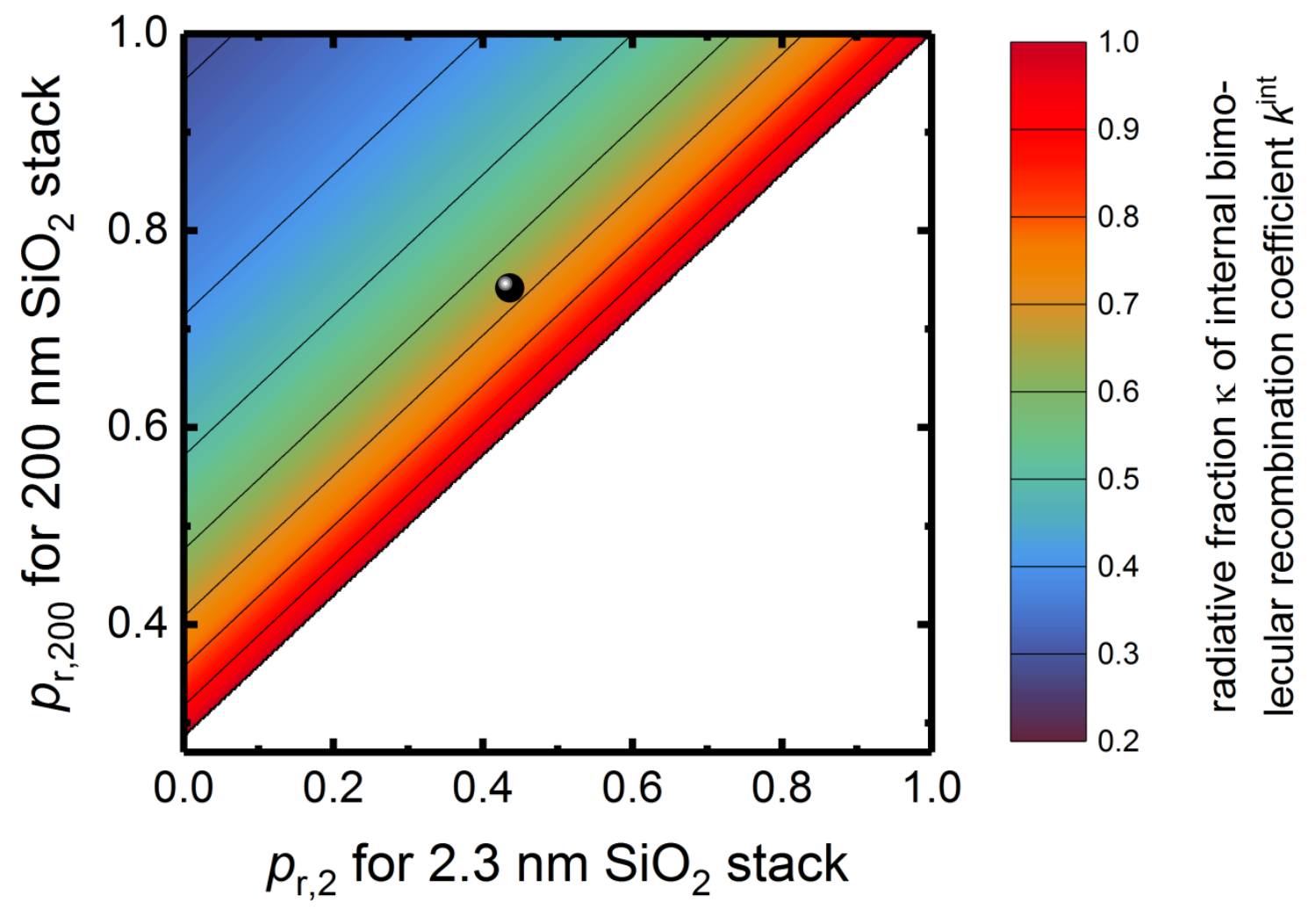

Figure 3. Simulated radiative fraction $\kappa$ of the bimolecular recombination coefficient $k^{\text {int }}$ according to Eq. (6) with a fixed $k_{\text {ratio }}^{\text {ext }}=1.4$ for a combination of photon recycling probabilities of the two layer stacks with different silicon dioxide thicknesses of $200 \mathrm{~nm}\left(p_{\mathrm{r}, 200}\right)$ and $2.3 \mathrm{~nm}$ $\left(p_{\mathrm{r}, 2}\right)$, respectively. The black sphere marks the situation of the best fit from the optical simulations resulting in $\kappa \approx 0.66$, when the amount of diffuse internal reflection at the rough perovskite/air interface becomes $p_{\text {diff }} \approx 0.7$.

Thus, we have to determine the photon recycling probabilities $p_{\mathrm{r}, 2}$ and $p_{\mathrm{r}, 200}$ in order to calculate the radiative fraction $\kappa$. As previously explained, internally emitted photons can either only escape, be parasitically absorbed or be reabsorbed. Thus by estimating the probability of 
emission $p_{\mathrm{e}}$ and parasitic absorption $p_{\mathrm{a}}$, we derive the probability of photon recycling $p_{\mathrm{r}}$ ultimately by $p_{\mathrm{r}}=1-p_{\mathrm{e}}-p_{\mathrm{a}}$. As shown in Ref. ${ }^{8}$, the outcoupling probability $p_{\mathrm{e}}$ is defined by

$$
p_{\mathrm{e}}=\frac{\varepsilon_{\mathrm{out}} \int_{0}^{\infty} A_{\mathrm{fsi}}(E) \phi_{\mathrm{bb}}(E) \mathrm{d} E}{\int_{0}^{\infty} \int 4 n_{\mathrm{r}, \text { pero }}^{2} \pi d \alpha(E) \phi_{\mathrm{bb}}(E) \mathrm{d} S \mathrm{~d} E},
$$

where the numerator stands for the outgoing photon flux calculated from the absorptance $A_{\mathrm{fsi}}(E)$ and the denominator for the total of the internal radiative recombination inside the absorber layer. ${ }^{8}$ In Eq. (7) the absorptance $A_{\mathrm{fsi}}(E)$ of the perovskite layer is defined for the illumination from the air side, here denoted as front side illumination. Furthermore, $\alpha(E)$ is the absorption coefficient, $\mathrm{d} S$ corresponds to a surface area element, $d$ refers to the thickness of the absorber layer and $\phi_{\mathrm{bb}}(E)$ is the black body radiation flux defined as

$$
\phi_{\mathrm{bb}}(E)=\frac{2 E^{2}}{\mathrm{~h}^{3} \mathrm{c}^{2}} \frac{1}{\exp \left[E /\left(k_{\mathrm{B}} T\right)-1\right]},
$$

with $\mathrm{h}$ being Planck's constant, $\mathrm{c}$ being the speed of light in vacuum. The Boltzmann constant is denoted as $k_{\mathrm{B}}$ and $T$ indicates the temperature of the sample. $\varepsilon_{\text {out }}$ in Eq. (7) denotes the étendue of outgoing light, which measures the "spread out" of light rays propagating through a surface element into a medium of refractive index $n_{\mathrm{r}, \text { out }}$ and are confined to a solid angle $\mathrm{d} \Omega$

$$
\mathrm{d} \varepsilon_{\text {out }}=n_{\mathrm{r}, \text { out }}^{2} \mathrm{~d} S \cos \theta \mathrm{d} \Omega .
$$

Consequently, photon emission into the hemisphere of air requires an outgoing étendue of $\mathrm{d} \varepsilon_{\text {out }}=\pi \mathrm{d} S$ and Eq. (7) becomes

$$
p_{\mathrm{e}}=\frac{\int_{0}^{\infty} A_{\mathrm{fsi}}(E) \phi_{\mathrm{bb}}(E) \mathrm{d} E}{\int_{0}^{\infty} 4 n_{\mathrm{r}, \text { pero }}^{2} d(E) \phi_{\mathrm{bb}}(E) \mathrm{d} E} .
$$


The probability of parasitic absorption is quite difficult to calculate in a general case. However, the samples shown in Fig. 1 are designed such that parasitic absorption is dominated by the light coupling into the Si substrate. Thus, for this specific case, we can calculate the probability of parasitic absorption in analogy to Eq. (10) by looking at the emission into the Si substrate and obtain

$$
p_{\mathrm{a}}=\frac{\int_{0}^{\infty} n_{\mathrm{r}, \mathrm{Si}}^{2} A_{\mathrm{bsi}}(E) \phi_{\mathrm{bb}}(E) \mathrm{d} E}{\int_{0}^{\infty} 4 n_{\mathrm{r}, \text { pero }}^{2} d(E) \phi_{\mathrm{bb}}(E) \mathrm{d} E} .
$$

In this case, the absorptance $A_{\mathrm{bsi}}(E)$ has to be simulated for radiation coupled into the perovskite film from the silicon halfspace; i. e. upon back side illumination. As stated before, with the knowledge of $p_{\mathrm{a}}$ and $p_{\mathrm{e}}$, the probability of photon recycling is found based on $p_{\mathrm{r}}=1-p_{\mathrm{e}}-p_{\mathrm{a}}$.

The absorptances needed in order to determine the various probabilities are calculated by a combination of a coherent transfer matrix method (TMM), ${ }^{40}$ which is valid for the simulation of flat layer stacks, and an incoherent light trapping (LT) approach according to analytical solutions derived by Green. ${ }^{41}$ Here, we take Lambertian light scattering at the perovskite/air interface into account, because this particular interface appears to be rough due to similar derived root-mean-square surface roughnesses of $R_{\mathrm{rms}, 200} \approx 45 \mathrm{~nm}$ and $R_{\mathrm{rms}, 2} \approx 51 \mathrm{~nm}$ from atomic force microscopy (AFM) images. Further information on the perovskite surface topography and the optical simulations can be found in the supporting information. By assuming that the absorptance in the absorber layer is obtained based on a combination of diffuse and specular internal reflection at the perovskite/air interface, we weight the corresponding absorptances by applying a haze factor $p_{\text {diff }}$ according to

$$
A\left(p_{\text {diff }}\right)=p_{\text {diff }} A_{\mathrm{LT}}+\left(1-p_{\text {diff }}\right) A_{\mathrm{TMM}},
$$


where $A_{\mathrm{LT}}$ and $A_{\mathrm{TMM}}$ are the absorptances derived by assuming ideal Lambertian light trapping and strictly flat interfaces based on TMM, respectively. Haze parameters are commonly used to describe scattering properties of e. g. textured glass/TCO substrates. ${ }^{42,43}$ In order to determine the relevant diffusive amount $p_{\text {diff }}$ in our samples, we use the absorptances $A_{\text {fsi }}\left(p_{\text {diff }}\right)$ from Eq. (12) to fit the measured PL spectra (emitted photon flux $\phi_{\mathrm{PL}}(E)$ ) according to ${ }^{44,45}$

$$
\phi_{\mathrm{PL}}(E) \propto A_{\mathrm{fsi}}(E) E^{2} \exp \left[-E /\left(k_{\mathrm{B}} T\right)\right] .
$$

The simulated PL spectra according to Eq. (13) are indicated in Fig. 4 as solid lines and match the experimental spectra best for $p_{\text {diff }} \approx 0.7$. The measured PL spectra of both samples are shown in Fig. 4 as green and red squares for the sample with the $200 \mathrm{~nm}$-thick and $2.3 \mathrm{~nm}$-thin silicon dioxide layer, respectively. A shift of the photoluminescence peak positions between the samples is visible. Additionally, the overall steady state PL intensity of the perovskite film on top of the $200 \mathrm{~nm}$-thick $\mathrm{SiO}_{2}$ layer is about 2.5 times higher than on top of the $2.3 \mathrm{~nm}$-thin $\mathrm{SiO}_{2}$ layer. This finding is also apparent from the TRPL data at $t=0$ in Fig. 2 (b). As the black body radiation is exponentially decreasing towards higher photon energies, the resulting emission flux $\phi_{\mathrm{PL}}(E)$ according to Eq. (13) is amplified for absorption in the small photon energy range. Due to varying absorption onsets of the samples (see supporting information for more details) according to different optical stack designs, the emission fluxes seen in Fig. 4 differ significantly in shape and intensity. 


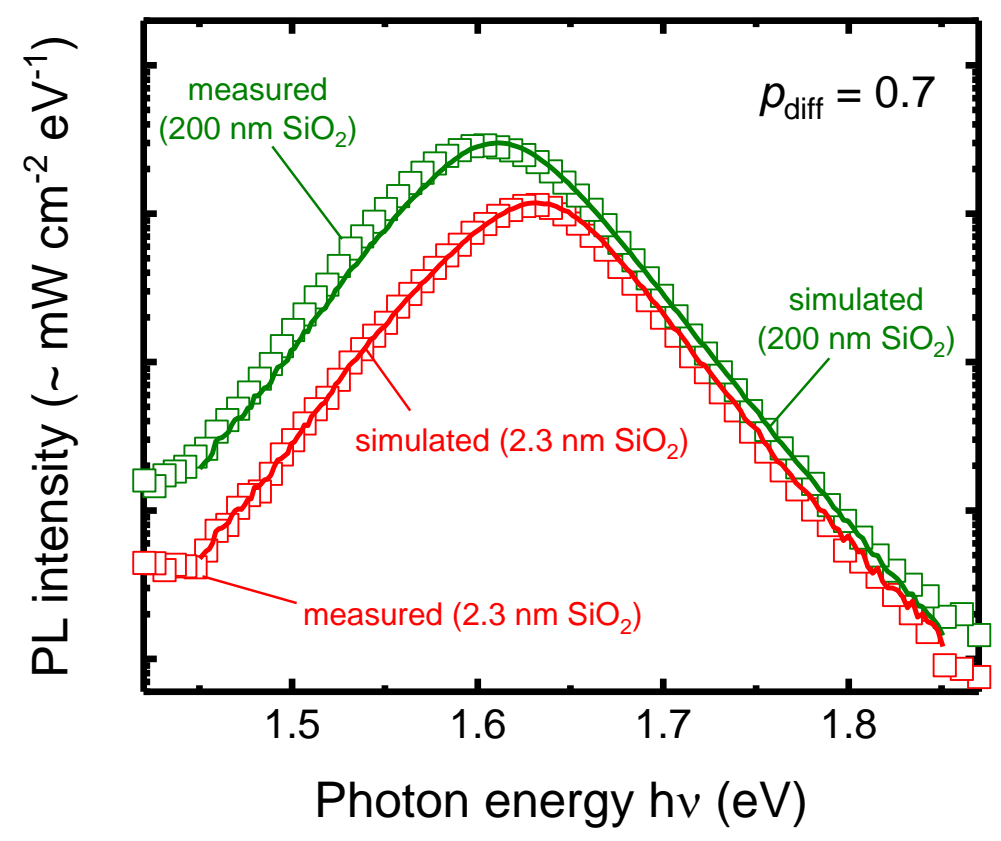

Figure 4. Measured steady state photoluminescence spectra of perovskite films coated on top of a $200 \mathrm{~nm}$-thick (green squares) and a $2.3 \mathrm{~nm}$-thin silicon dioxide layer (red squares). The solid lines represent simulations of the spectra assuming a fraction of internal diffuse light scattering of $p_{\text {diff }}=0.7$ at the air/perovskite interface.

Identifying a perovskite surface roughness of $R_{\mathrm{rms}}=50 \mathrm{~nm}$, van Eerden et al ${ }^{46}$ rely on a similar diffuse share of $p_{\text {diff }}=0.5$ in their optical calculations. The resulting probabilities for photon recycling, parasitic absorption and outcoupling derived from Eq. (10) and (11) are indicated in Fig. 1 for $p_{\text {diff }} \approx 0.7$. Finally, the obtained photon recycling probabilities of $p_{\mathrm{r}, 200} \approx$ 0.74 and $p_{\mathrm{r}, 2} \approx 0.44$ result in a radiative fraction of $\kappa \approx 0.66$ (see black sphere in Fig. 3), i. e. a significant amount of non-radiative bimolecular recombination is revealed.

We note that the existence of a non-radiative bimolecular recombination channel already sets an upper limit of $66 \%$ to the internal luminescence quantum efficiency, which is in direct 
contrast to findings derived from laser cooling experiments on perovskite single crystals, where Ha et al. ${ }^{47}$ report external luminescence quantum efficiencies well above $90 \%$. Therefore, questions arise about the origin of the non-radiative bimolecular recombination mechanism and to what extent the sample type has an influence, e. g. in the form of significantly different amounts of defects present in perovskite thin films and single crystals, respectively. ${ }^{48-52}$ Richter et al. ${ }^{31}$ propose trap-assisted Auger recombination processes as possible cause of non-radiative bimolecular recombination in lead halide perovskites. These mechanisms involve transitions between two free charge carriers and an unoccupied trap or a bound carrier, respectively, and ultimately appear bimolecular. ${ }^{53-55}$ Consequently, trap-assisted Auger recombination as possible non-radiative bimolecular decay channel would be more relevant for polycrystalline thin films than for pure single crystals assuming that the latter have lower defect densities. This finding could be a possible explanation of the significant differences in the obtained luminescence quantum efficiencies.

In conclusion, time-resolved photoluminescence measurements have demonstrated that the externally measured radiative decay rates and photon recycling probabilities in metal halide perovskite films can be controlled by the optical design of the substrate layers. Different designs have been realized by different thicknesses of a thermally grown oxide on a silicon wafer warranting that the growth and the structural properties of the metal halide perovskite films are the same for each type of substrate. The latter fact is proven by nearly identical SRH lifetimes for both films. In contrast, varying external decay rates unveil the fundamental physical fact that external radiative decay rates and their difference to the internal radiative recombination rate are controlled by optics alone. Optical simulations based on a combination of coherent and incoherent effects consistently explain the dependence of emission spectra and decay rates on the 
$\mathrm{SiO}_{2}$ thickness. Furthermore, the comparison between experiment and simulation suggests that the internal bimolecular recombination coefficient $k^{\text {int }}$ (intrinsic parameter without photon recycling events) is not $100 \%$ radiative but contains a non-radiative contribution of about (1$\kappa) \approx 34 \%$, which is close to the value $(1-\kappa) \approx 24 \%$ for $\mathrm{MAPI}_{3-\mathrm{x}} \mathrm{Cl}_{\mathrm{x}}$ reported by Richter $e t$ $a l .{ }^{31}$ Such a non-radiative bimolecular recombination channel affects the limiting radiative efficiency of metal halide perovskites, because it will reduce the maximum efficiency of perovskite solar cells. While there is currently no clear understanding of the origin of this nonradiative bimolecular recombination mechanism, it should be one focus of future investigations on the metal-halide perovskite material system. 


\section{ACKNOWLEDGMENT}

The authors acknowledge support from the Deutsche Forschungsgemeinschaft (DFG) (Grant No.

KI-1571/2-1 and RA-473/7-1). We thank Malte Köhler for providing the silicon substrates, Irina Kühn for perovskite layer fabrication and Markus Hülsbeck for assistance with TRPL and PL measurements. Additionally, we acknowledge Pascal Foucart for AFM measurements and Pascal Kaienburg for SEM measurements.

\section{SUPPORTING INFORMATION}

Experimental methods, details for simulating PL transients, detailed description of the optical simulations, morphology of perovskite layers 


\section{REFERENCES}

(1) van Roosbroeck, W.; Shockley, W. Photon-Radiative Recombination of Electrons and Holes in Germanium. Phys. Rev. 1954, 94, 1558-1560.

(2) Martí, A.; Balenzategui, J. L.; Reyna, R. F. Photon Recycling and Shockley's Diode Equation. J. Appl. Phys. 1997, 82, 4067-4075.

(3) Shockley, W.; Queisser, H. J. Detailed Balance Limit of Efficiency of $p$-n Junction Solar Cells. J. Appl. Phys. 1961, 32, 510-519.

(4) Schnitzer, I.; Yablonovitch, E.; Caneau, C.; Gmitter, T. J. Ultrahigh Spontaneous Emission Quantum Efficiency, 99.7\% Internally and 72\% Externally, from AlGaAs/GaAs/AlGaAs Double Heterostructures. Appl. Phys. Lett. 1993, 62, 131-133.

(5) Miller, O. D.; Yablonovitch, E.; Kurtz, S. R. Strong Internal and External Luminescence as Solar Cells Approach the Shockley-Queisser Limit. IEEE J. Photovolt. 2012, 2, 303311.

(6) Yablonovitch, E.; Miller, O. D.; Kurtz, S. R. The Opto-Electronic Physics That Broke the Efficiency Limit in Solar Cells. In 38th IEEE Photovoltaic Specialists Conference (PVSC); 2012; pp 001556-001559.

(7) Mattheis, J.; Werner, J. H.; Rau, U. Finite Mobility Effects on the Radiative Efficiency Limit of pn-Junction Solar Cells. Phys. Rev. B 2008, 77, 085203.

(8) Rau, U.; Paetzold, U. W.; Kirchartz, T. Thermodynamics of Light Management in Photovoltaic Devices. Phys. Rev. B 2014, 90, 035211.

(9) deQuilettes, D. W.; Koch, S.; Burke, S.; Paranji, R. K.; Shropshire, A. J.; Ziffer, M. E.; Ginger, D. S. Photoluminescence Lifetimes Exceeding $8 \mu \mathrm{s}$ and Quantum Yields Exceeding 30\% in Hybrid Perovskite Thin Films by Ligand Passivation. ACS Energy Lett. 2016, 1, 438-444.

(10) Bi, Y.; Hutter, E. M.; Fang, Y.; Dong, Q.; Huang, J.; Savenije, T. J. Charge Carrier Lifetimes Exceeding $15 \mu$ s in Methylammonium Lead Iodide Single Crystals. J. Phys. Chem. Lett. 2016, 7, 923-928.

(11) Sutter-Fella, C. M.; Li, Y.; Amani, M.; Ager, J. W.; Toma, F. M.; Yablonovitch, E.; Sharp, I. D.; Javey, A. High Photoluminescence Quantum Yield in Band Gap Tunable Bromide Containing Mixed Halide Perovskites. Nano Lett. 2016, 16, 800-806.

(12) Tvingstedt, K.; Malinkiewicz, O.; Baumann, A.; Deibel, C.; Snaith, H. J.; Dyakonov, V.; Bolink, H. J. Radiative Efficiency of Lead Iodide Based Perovskite Solar Cells. Sci. Rep. 2014, 4, 6071.

(13) Tress, W.; Marinova, N.; Inganäs, O.; Nazeeruddin, M. K.; Zakeeruddin, S. M.; Grätzel, M. Predicting the Open-Circuit Voltage of $\mathrm{CH}_{3} \mathrm{NH}_{3} \mathrm{PbI}_{3}$ Perovskite Solar Cells Using Electroluminescence and Photovoltaic Quantum Efficiency Spectra: The Role of Radiative and Non-Radiative Recombination. Adv. Energy Mater. 2015, 5, 1400812.

(14) Yao, J.; Kirchartz, T.; Vezie, M. S.; Faist, M. A.; Gong, W.; He, Z.; Wu, H.; Troughton, J.; Watson, T.; Bryant, D.; et al. Quantifying Losses in Open-Circuit Voltage in SolutionProcessable Solar Cells. Phys. Rev. Appl. 2015, 4, 014020.

(15) Pazos-Outón, L. M.; Szumilo, M.; Lamboll, R.; Richter, J. M.; Crespo-Quesada, M.; AbdiJalebi, M.; Beeson, H. J.; Vrućinić, M.; Alsari, M.; Snaith, H. J.; et al. Photon Recycling in Lead Iodide Perovskite Solar Cells. Science 2016, 351, 1430-1433. 
(16) Saliba, M.; Zhang, W.; Burlakov, V. M.; Stranks, S. D.; Sun, Y.; Ball, J. M.; Johnston, M. B.; Goriely, A.; Wiesner, U.; Snaith, H. J. Plasmonic-Induced Photon Recycling in Metal Halide Perovskite Solar Cells. Adv. Funct. Mater. 2015, 25, 5038-5046.

(17) Sha, W. E. I.; Ren, X.; Chen, L.; Choy, W. C. H. The Efficiency Limit of $\mathrm{CH}_{3} \mathrm{NH}_{3} \mathrm{PbI}_{3}$ Perovskite Solar Cells. Appl. Phys. Lett. 2015, 106, 221104.

(18) Fang, Y.; Wei, H.; Dong, Q.; Huang, J. Quantification of Re-Absorption and Re-Emission Processes to Determine Photon Recycling Efficiency in Perovskite Single Crystals. Nat. Commun. 2017, 8, 14417.

(19) Yamada, T.; Yamada, Y.; Nakaike, Y.; Wakamiya, A.; Kanemitsu, Y. Photon Emission and Reabsorption Processes in $\mathrm{CH}_{3} \mathrm{NH}_{3} \mathrm{PbBr}_{3}$ Single Crystals Revealed by Time-Resolved Two-Photon-Excitation Photoluminescence Microscopy. Phys. Rev. Appl. 2017, 7, 014001.

(20) Kanemitsu, Y. Luminescence Spectroscopy of Lead-Halide Perovskites: Materials Properties and Application as Photovoltaic Devices. J Mater Chem C 2017, 5, 3427-3437.

(21) Stern, F.; Woodall, J. M. Photon Recycling in Semiconductor Lasers. J. Appl. Phys. 1974, 45, 3904-3906.

(22) Asbeck, P. Self-Absorption Effects on the Radiative Lifetime in GaAs-GaAlAs Double Heterostructures. J. Appl. Phys. 1977, 48, 820-822.

(23) Ahrenkiel, R. K.; Dunlavy, D. J.; Keyes, B.; Vernon, S. M.; Dixon, T. M.; Tobin, S. P.; Miller, K. L.; Hayes, R. E. Ultralong Minority-Carrier Lifetime Epitaxial GaAs by Photon Recycling. Appl. Phys. Lett. 1989, 55, 1088-1090.

(24) Ahrenkiel, R. K.; Keyes, B. M.; Lush, G. B.; Melloch, M. R.; Lundstrom, M. S.; MacMillan, H. F. Minority-carrier Lifetime and Photon Recycling in $n$-GaAs. J. Vac. Sci. Technol. A: Vac. Surf. Films 1992, 10, 990-995.

(25) Staub, F.; Hempel, H.; Hebig, J.-C.; Mock, J.; Paetzold, U. W.; Rau, U.; Unold, T.; Kirchartz, T. Beyond Bulk Lifetimes: Insights into Lead Halide Perovskite Films from Time-Resolved Photoluminescence. Phys. Rev. Appl. 2016, 6, 044017.

(26) Steiner, M. A.; Geisz, J. F.; García, I.; Friedman, D. J.; Duda, A.; Kurtz, S. R. Optical Enhancement of the Open-Circuit Voltage in High Quality GaAs Solar Cells. J. Appl. Phys. 2013, 113, 123109.

(27) Kirchartz, T.; Staub, F.; Rau, U. Impact of Photon Recycling on the Open-Circuit Voltage of Metal Halide Perovskite Solar Cells. ACS Energy Lett. 2016, 1, 731-739.

(28) Stranks, S. D.; Burlakov, V. M.; Leijtens, T.; Ball, J. M.; Goriely, A.; Snaith, H. J. Recombination Kinetics in Organic-Inorganic Perovskites: Excitons, Free Charge, and Subgap States. Phys. Rev. Appl. 2014, 2, 034007.

(29) Wehrenfennig, C.; Eperon, G. E.; Johnston, M. B.; Snaith, H. J.; Herz, L. M. High Charge Carrier Mobilities and Lifetimes in Organolead Trihalide Perovskites. Adv. Mater. 2014, 26, 1584-1589.

(30) Hutter, E. M.; Eperon, G. E.; Stranks, S. D.; Savenije, T. J. Charge Carriers in Planar and Meso-Structured Organic-Inorganic Perovskites: Mobilities, Lifetimes, and Concentrations of Trap States. J. Phys. Chem. Lett. 2015, 6, 3082-3090.

(31) Richter, J. M.; Abdi-Jalebi, M.; Sadhanala, A.; Tabachnyk, M.; Rivett, J. P. H.; PazosOutón, L. M.; Gödel, K. C.; Price, M.; Deschler, F.; Friend, R. H. Enhancing Photoluminescence Yields in Lead Halide Perovskites by Photon Recycling and Light Out-Coupling. Nat. Commun. 2016, 7, 13941. 
(32) Herzinger, C. M.; Johs, B.; McGahan, W. A.; Woollam, J. A.; Paulson, W. Ellipsometric Determination of Optical Constants for Silicon and Thermally Grown Silicon Dioxide via a Multi-Sample, Multi-Wavelength, Multi-Angle Investigation. J. Appl. Phys. 1998, 83, 3323-3336.

(33) Löper, P.; Stuckelberger, M.; Niesen, B.; Werner, J.; Filipič, M.; Moon, S.-J.; Yum, J.-H.; Topič, M.; De Wolf, S.; Ballif, C. Complex Refractive Index Spectra of $\mathrm{CH}_{3} \mathrm{NH}_{3} \mathrm{PbI}_{3}$ Perovskite Thin Films Determined by Spectroscopic Ellipsometry and Spectrophotometry. J. Phys. Chem. Lett. 2015, 6, 66-71.

(34) Suárez, I.; Juárez-Pérez, E. J.; Bisquert, J.; Mora-Seró, I.; Martínez-Pastor, J. P. Polymer/Perovskite Amplifying Waveguides for Active Hybrid Silicon Photonics. Adv. Mater. 2015, 27, 6157-6162.

(35) Aspnes, D. E.; Studna, A. A. Dielectric Functions and Optical Parameters of Si, Ge, GaP, GaAs, GaSb, InP, InAs, and InSb from 1.5 to 6.0 eV. Phys. Rev. B 1983, 27, 985-1009.

(36) Marin-Beloqui, J. M.; Hernández, J. P.; Palomares, E. Photo-Induced Charge Recombination Kinetics in $\mathrm{MAPbI}_{3-\mathrm{x}} \mathrm{Cl}_{\mathrm{x}}$ Perovskite-like Solar Cells Using Low Band-Gap Polymers as Hole Conductors. Chem Commun 2014, 50, 14566-14569.

(37) Ponseca Jr., C. S.; Hutter, E. M.; Piatkowski, P.; Cohen, B.; Pascher, T.; Douhal, A.; Yartsev, A.; Sundström, V.; Savenije, T. J. Mechanism of Charge Transfer and Recombination Dynamics in Organo Metal Halide Perovskites and Organic Electrodes, PCBM, and Spiro-OMeTAD: Role of Dark Carriers. J. Am. Chem. Soc. 2015, 137, 1604316048.

(38) Brauer, J. C.; Lee, Y. H.; Nazeeruddin, M. K.; Banerji, N. Charge Transfer Dynamics from Organometal Halide Perovskite to Polymeric Hole Transport Materials in Hybrid Solar Cells. J. Phys. Chem. Lett. 2015, 6, 3675-3681.

(39) Yamada, Y.; Nakamura, T.; Endo, M.; Wakamiya, A.; Kanemitsu, Y. Photocarrier Recombination Dynamics in Perovskite $\mathrm{CH}_{3} \mathrm{NH}_{3} \mathrm{PbI}_{3}$ for Solar Cell Applications. J. Am. Chem. Soc. 2014, 136, 11610-11613.

(40) Byrnes, S. J. Multilayer Optical Calculations. arXiv:1603.02720v2. 2016.

(41) Green, M. A. Lambertian Light Trapping in Textured Solar Cells and Light-Emitting Diodes: Analytical Solutions. Prog. Photovolt. Res. Appl. 2002, 10, 235-241.

(42) Zeman, M.; van Swaaij, R. A. C. M. M.; Metselaar, J. W.; Schropp, R. E. I. Optical Modeling of $a$-Si:H Solar Cells with Rough Interfaces: Effect of Back Contact and Interface Roughness. J. Appl. Phys. 2000, 88, 6436-6443.

(43) Krč, J.; Zeman, M.; Smole, F.; Topič, M. Optical Modeling of $a$-Si:H Solar Cells Deposited on Textured Glass/SnO2 Substrates. J. Appl. Phys. 2002, 92, 749-755.

(44) Trupke, T.; Daub, E.; Würfel, P. Absorptivity of Silicon Solar Cells Obtained from Luminescence. Sol. Energy Mater. Sol. Cells 1998, 53, 103-114.

(45) Trupke, T.; Green, M. A.; Würfel, P.; Altermatt, P. P.; Wang, A.; Zhao, J.; Corkish, R. Temperature Dependence of the Radiative Recombination Coefficient of Intrinsic Crystalline Silicon. J. Appl. Phys. 2003, 94, 4930-4937.

(46) van Eerden, M.; Jaysankar, M.; Hadipour, A.; Merckx, T.; Schermer, J. J.; Aernouts, T.; Poortmans, J.; Paetzold, U. W. Optical Analysis of Planar Multicrystalline Perovskite Solar Cells. Adv. Opt. Mater. 2017, 1700151.

(47) Ha, S.-T.; Shen, C.; Zhang, J.; Xiong, Q. Laser Cooling of Organic-Inorganic Lead Halide Perovskites. Nat. Photonics 2015, 10, 115-121. 
(48) Duan, H.-S.; Zhou, H.; Chen, Q.; Sun, P.; Luo, S.; Song, T.-B.; Bob, B.; Yang, Y. The Identification and Characterization of Defect States in Hybrid Organic-Inorganic Perovskite Photovoltaics. Phys. Chem. Chem. Phys. 2015, 17, 112-116.

(49) Baumann, A.; Väth, S.; Rieder, P.; Heiber, M. C.; Tvingstedt, K.; Dyakonov, V. Identification of Trap States in Perovskite Solar Cells. J. Phys. Chem. Lett. 2015, 6, 2350 2354.

(50) Shi, D.; Adinolfi, V.; Comin, R.; Yuan, M.; Alarousu, E.; Buin, A.; Chen, Y.; Hoogland, S.; Rothenberger, A.; Katsiev, K.; et al. Low Trap-State Density and Long Carrier Diffusion in Organolead Trihalide Perovskite Single Crystals. Science 2015, 347, 519522.

(51) Dong, Q.; Fang, Y.; Shao, Y.; Mulligan, P.; Qiu, J.; Cao, L.; Huang, J. Electron-Hole Diffusion Lengths $>175 \mu \mathrm{m}$ in Solution-Grown $\mathrm{CH}_{3} \mathrm{NH}_{3} \mathrm{PbI}_{3}$ Single Crystals. Science 2015, 347, 967-970.

(52) Saidaminov, M. I.; Abdelhady, A. L.; Murali, B.; Alarousu, E.; Burlakov, V. M.; Peng, W.; Dursun, I.; Wang, L.; He, Y.; Maculan, G.; et al. High-Quality Bulk Hybrid Perovskite Single Crystals within Minutes by Inverse Temperature Crystallization. Nat. Commun. 2015, 6, 7586.

(53) Landsberg, P. T.; Rhys-Roberts, C.; Lal, P. Auger Recombination and Impact Ionization Involving Traps in Semiconductors. Proc. Phys. Soc. 1964, 84, 915-931.

(54) Landsberg, P. T.; Adams, M. J. Radiative and Auger Processes in Semiconductors. $J$. Lumin. 1973, 7, 3-34.

(55) Stoneham, A. M. The Auger Effect and Related Phenomena. In Theory of Defects in Solids: Electronic Structure and Defects in Insulators and Semiconductors; Clarendon Press: Oxford, 1975; pp 539-546. 